\title{
GESTANTES DE EDAD MATERNA AVANZADA PRESENTAN MAYOR RIESGO MATERNO PERINATAL QUE LAS GESTANTES ADOLESCENTES
}

\author{
Félix Dasio Ayala-Peralta ${ }^{1}$, Odalis Alejandra Berrocal Montejo², Enrique Guevara Ríos ${ }^{3}$, \\ Antonio Luna Figueroa ${ }^{4}$, César Carranza Asmat ${ }^{5}$, Oscar Antonio Limay Ríos ${ }^{6}$
}

\begin{abstract}
RESUMEN
Objetivo: Determinar si la gestación en edad materna avanzada está asociada a mayor riesgo de complicaciones materno perinatales que en la gestación adolescente. Materiales y métodos: Se planteó un estudio observacional, correlacional, retrospectivo, transversal, con una población de 6475 gestantes añosas y 7645 gestantes adolescentes atendidas en el Instituto Nacional Materno Perinatal que se han controlado y culminado parto en la institución en el periodo comprendido entre enero del 2012 a diciembre de 2015. Resultados: Las complicaciones más frecuentes en gestantes añosas fueron en primer lugar preeclampsia, seguida de anemia y RPM. En cuanto a gestantes adolescentes las más frecuentes fueron en orden de aparición: RPM, anemia y preeclampsia. Se observó asociación entre el riesgo de complicaciones maternas con las gestantes de edad materna avanzada, así como también se encontró que no existe asociación entre las edades materna avanzada y la morbilidad perinatal. Conclusiones: Se halló que el grupo de gestantes con mayor riesgo de presentar complicaciones maternas fue el de edad materna avanzada con un OR de 1.08 en comparación a las adolescentes.
\end{abstract}

Palabras clave: Edad materna avanzada; Adolescente; Complicación materno perinatal (Fuente DeCS BIREME).

\section{PREGNANT WOMEN OF ADVANCED MATERNAL AGE PRESENT HIGHER RISK PERINATAL MATERNAL THAN ADOLESCENT PREGNANT WOMEN}

\begin{abstract}
Objective: determine if gestation in advanced maternal age is associated with a higher risk of maternal perinatal complications than in adolescent gestation. Materials and methods: observational, correlational, retrospective, cross-sectional study was carried out with a population of 6475 pregnant women and 7645 pregnant women attended at the National Maternal Perinatal Institute who have been controlled and culminated in the institution in the period between January From 2012 to December 2015. Results: The most frequent complications in aged pregnant women were in the first place preeclampsia, followed by anemia and RPM. As for adolescent pregnant women the most frequent were in order of appearance: RPM, anemia and preeclampsia. It was observed an association between the risk of maternal complications with pregnant women of advanced maternal age, as well as it was found that there is no association between advanced maternal ages and perinatal morbidity. Conclusions: It was found that the group of pregnant women most at risk of presenting maternal complications was the advanced maternal age with an OR of 1.08 compared to the adolescents.
\end{abstract}

Keywords: Advanced maternal age; Teen; Maternal perinatal complication (Source: MeSH NLM).

\section{INTRODUCCIÓN}

La maternidad en las edades extremas de la vida reproductiva últimamente es objeto de estudio, ya que las tasas de fecundidad en ambos grupos han ido aumentando a lo largo de los años, y junto con ello se ha demostrado que en ambos grupos, la edad materna es un factor de riesgo para la presentación de complicaciones relacionadas con el embarazo, comprometiendo en distinto grado a la madre y al niño ${ }^{1-4}$.
Por diversos motivos, las mujeres hoy en día se inician en la maternidad tardíamente y nuestro país ya en vías de desarrollo presenta en la actualidad un incremento en la frecuencia de mujeres gestantes de edad materna avanzada, esto se encuentra probablemente relacionado a diversos factores, como la necesidad de tener una adecuada condición económica o tal vez una relación estable, y esto se ve reflejado en las atenciones prenatales y partos en mujeres de edad cada vez mayor ${ }^{5,6}$.

Médico GínecoObstetra. Magíster en Salud Reproductiva. Profesor de la Facultad de Medicina Humana de la Universidad San Martín de Porres y de Ciencias de la Salud de la Universidad Norbert Wiener. Jefe del Servicio de Climaterio y Menopausia del Instituto Nacional Materno Perinatal. Miembro de la Red Latinoamericana de Investigadores en Climaterio (REDLINC). Lima-Perú.

Estudiante de la Facultad de Medicina Humana de la Universidad San Juan Bautista. Lima-Perú

3 Médico Ginecólogo Obstetra, Director del Instituto Nacional Materno Perinatal, Coordinador de Asistencia Técnica del Instituto de Salud Popular, Consultor de Pathfinder International, Docente de la Universidad Nacional Mayor de San Marcos, Lima, Perú / Director-in-Chief Instituto Nacional Materno Perinatal, Technical Assistance Cooordinator Instituto de Salud Popular, Consultor Pathfinder International, Docente Universidad Nacional Mayor de San Marcos, Lima, Perú.

4 Médico GínecoObstetra. Profesor Principal de la Universidad Nacional Mayor de San Marcos. Director Ejecutivo de Investigación, Docencia y Atención en Obstetricia y Ginecología del Instituto Nacional Materno Perinatal. Lima-Perú.

5 Médico GínecoObstetra. Profesor Asociadol de la Universidad Nacional Mayor de San Marcos. Director Ejecutivo de la Oficina de Investigación y Docencia Especializada. Instituto Nacional Materno Perinatal. Lima-Perú.

6 Médico Gíneco-Obstetra. Jefe de Unidad de Investigación y Asistente del Servicio de Medicina Fetal del Instituto Nacional Materno Perinatal. Lima-Perú.

Recibido: 01-12-16 Aprobado: 30-06-17 
Diversos estudios muestran que las complicaciones materno perinatales que se presentan con mayor frecuencia durante y después de la gestación son los trastornos hipertensivos gestacionales, diabetes gestacional, desgarros perineales en la gestante; y por otro lado en el recién nacido retardo del crecimiento intrauterino, prematuridad, bajo peso al nacer y óbito fetal ${ }^{7}$.

Se pretende demostrar que la gestante de edad materna avanzada presenta mayor riesgo de complicaciones materno perinatales frente a la gestante adolescente, otro grupo de alto riesgo para presentar estas complicaciones; y así poder trabajar en acciones que aseguren el proceso desde el inicio de la gestación, para lograr una detección oportuna de eventos adversos y control de los mismos.

\section{MATERIALES Y MÉTODOS}

El presente estudio es de tipo cuantitativo, observacional, retrospectivo, transversal, correlacional; fue realizado en el Instituto Nacional Materno Perinatal (INMP) ubicado en la ciudad de Lima entre el año 2012 y 2015. La población estuvo constituida por el universo de 6475 gestantes añosas y 7645 gestantes adolescentes que han sido controladas y culminado parto en el INMP, que cumplieron los siguientes criterios de inclusión: gestantes de edad materna avanzada (de 35 años a más) que se controlaron y culminaron el parto en la institución, gestantes adolescentes que se controlaron y culminaron el parto en la institución, gestantes adolescentes y añosas con registro de la madre y del recién nacido. No se incluyó en el estudio a gestantes en presencia de comorbilidades y con gestación múltiple.

Los datos se registraron en una ficha técnica. La información se obtuvo en base a los registros de nacimientos, registro de egresos, y registros de hospitalización de neonatología del INMP. Estos datos fueron utilizados para la elaboración de una ficha de recolección de datos con las pacientes que cumplían los criterios de inclusión y exclusión en base a los juicios de expertos y de la bibliografía consultada, el mismo que fue validado antes de aplicarlo.

La información obtenida se tabulo en base de datos de Microsoft Office Excel 2013 de acuerdo a la ficha de recolección de datos, para luego realizar el análisis con el programa estadístico SPSS versión 12,0 para Windows.

Para analizar la posible asociación entre la edad materna y las morbilidades maternas y perinatales se empleó la prueba Chi cuadrado $x^{2}$, con un intervalo de confianza del $95 \%$. El valor $p$ $<0,05$ fue considerado para la significancia estadística.

\section{RESULTADOS}

Se observó que de 7645 adolescentes, el 79,2\% (6053 pacientes) no presento complicaciones maternas y un

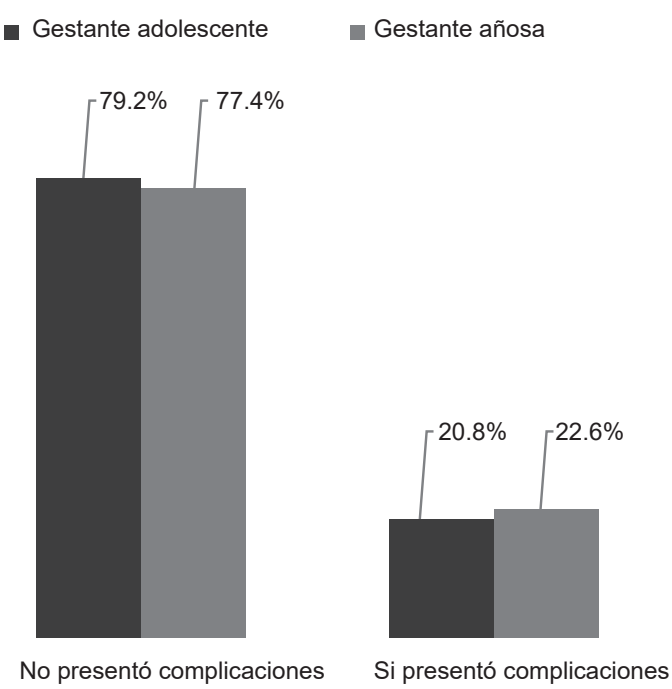

Gráfico 1. Presentación de Complicaciones maternas en la gestante adolescente y añosa.

Fuente: Ficha de Recolección de datos.

20,8\%(1592 pacientes) si presentó. En el grupo de gestantes añosas tenemos a 6475 pacientes de las cuales el $77,4 \%(5012$ pacientes) no presento complicaciones maternas, y un 22,6\%(1463 pacientes) que si presentó (Gráfico1).

De 1592 gestantes adolescentes que si presentaron complicaciones maternas el 6,33\% (484 pacientes) presentaron ruptura prematura de membranas; un 4,51\% (345 pacientes) presentó anemia; el 3,1\% (237 pacientes) tuvieron preeclampsia; Infecciones del tracto urinario con 2,21\% (169 pacientes); y en quinto lugar distocia de presentación con 1,2\% (92 pacientes). Las demás complicaciones presentan mínimo valor porcentual menor a 1\%. De 1463 gestantes añosas que si presentaron complicaciones maternas el $4,65 \%$ (301 pacientes) presentó preeclampsia; 4,62\% (299 pacientes) presentaron anemia; ruptura prematura de membranas con 4,57\% (296 pacientes); infecciones del tracto urinario con 2,3\% (149 pacientes); en quinto lugar placenta previa con un $1,27 \%$ (82 pacientes); distocia de presentación en 1,25\% (81 pacientes). Las demás complicaciones con mínimo valor pocentual menor a $1 \%$ (Tabla 1y 2).

En cuanto a complicaciones perinatales, se observa que de 7645 gestantes adolescentes, el 97,7\% (7086) no presento complicaciones y un 7,3\%(559 pacientes) si presentó. En el grupo de gestantes añosas tenemos a 6475 pacientes de las cuales el 92,4\%(492 pacientes) no presento complicaciones perinatales, y un 7,6\%(492 pacientes) que si presentó (Gráfico 2).

De 7645 gestantes adolescentes, el 4,81\% (368 pacientes) presentaron sufrimiento fetal agudo; 2,09\% (160 pacientes); hipoglicemia del recién nacido con $0,17 \%$ (13 pacientes); al 
Tabla 1.Complicaciones maternas en la gestante adolescente

\begin{tabular}{|l|r|r|}
\hline \multicolumn{1}{|c|}{ COMPIICACION MATERNA } & FRECUENCIA & PORCENTAJE \\
\hline SIN COMPLICACIONES & 6053 & 79.18 \\
\hline RUPTURA PREMATURA DE MEMBRANAS & 484 & 6.33 \\
\hline ANEMIA & 345 & 4.51 \\
\hline PREECLAMPSIA & 237 & 3.10 \\
\hline INFECCION DEL TRACTO URINARIO & 169 & 2.21 \\
\hline DISTOCIA DE PRESENTACION & 92 & 1.20 \\
\hline ALUMBRAMIENTO INCOMPLETO & 59 & 0.77 \\
\hline AMENAZA DE PARTO PRE TERMINO & 54 & 0.71 \\
\hline HEMORRAGIA POST PARTO & 31 & 0.41 \\
\hline DESGARRO PERINEAL & 25 & 0.33 \\
\hline DESPRENDIMIENTO PREMATURO DE PLACENTA & 16 & 0.21 \\
\hline TRABAJO DE PARTO PRE TERMINO & 15 & 0.20 \\
\hline ABORTO & 14 & 0.18 \\
\hline SOBREPESO, OBESIDAD, OBESIDAD MORBIDA & 11 & 0.14 \\
\hline HIPEREMESIS & 10 & 0.13 \\
\hline MUERTE MATERNA & 7 & 0.09 \\
\hline PLACENTA PREVIA & 7 & 0.09 \\
\hline AMENAZA DE ABORTO & 6 & 0.08 \\
\hline DIABETES GESTACIONAL & 5 & 0.07 \\
\hline ECLAMPSIA & 4 & 0.05 \\
\hline S. HELLP & 1 & 0.01 \\
\hline Total & 7645 & 100.00 \\
\hline
\end{tabular}

Fuente: Ficha de Recolección de datos
- Gestante añosa

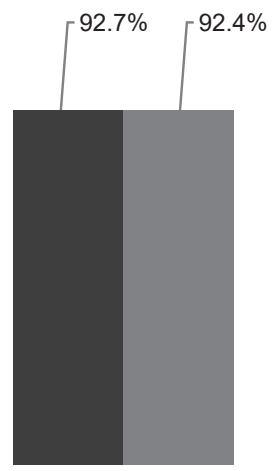

No presentó complicaciones

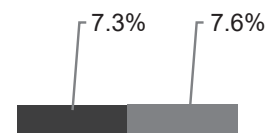

Si presentó complicaciones
Gráfico 2. Presentación de complicaciones perinatales en la gestante adolescente y añosa.

Fuente: Ficha de Recolección de datos.

Tabla 2.Complicaciones maternas en la gestante añosa

\begin{tabular}{|l|r|r|}
\hline COMPLICACIONES MATERNAS & $\begin{array}{l}\text { FRECUENCI } \\
\text { A }\end{array}$ & PORCENTAIE \\
\hline SIN COMPLICACIONES & 5012 & 77.41 \\
\hline PREECLAMPSIA & 301 & 4.65 \\
\hline ANEMIA & 299 & 4.62 \\
\hline RUPTURA PREMATURA DE MEMBRANAS & 296 & 4.57 \\
\hline ITU & 149 & 2.30 \\
\hline PLACENTA PREVIA & 82 & 1.27 \\
\hline DISTOCIA DE PRESENTACION & 81 & 1.25 \\
\hline ABORTO & 44 & 0.68 \\
\hline AMENAZA DE PARTO PRETERMINO & 44 & 0.68 \\
\hline ALUMBRAMIENTO INCOMPLETA & 42 & 0.65 \\
\hline SOBREPESO, OBESIDAD, OBESIDAD MORBIDA & 34 & 0.53 \\
\hline DESPRENDIMIENTO PREMATURO DE PLACENTA & 25 & 0.39 \\
\hline AMENAZA DE ABORTO & 18 & 0.28 \\
\hline HEMORRAGIA POST PARTO & 12 & 0.19 \\
\hline DESGARRO PERINEAL & 11 & 0.17 \\
\hline TRABAJO DE PARTO PRETERMINO & 9 & 0.14 \\
\hline HIPEREMESIS & 4 & 0.06 \\
\hline S. DE HELLP & 4 & 0.06 \\
\hline MUERTE MATERNA & 4 & 0.06 \\
\hline ECLAMPSIA & 3 & 0.05 \\
\hline DIABETES MELLITUS GESTACIONAL & 1 & 0.02 \\
\hline & 6475 & 100.00 \\
\hline
\end{tabular}

Fuente: Ficha de Recolección de datos

igual que óbito fetal en un $0.17 \%$ (13 pacientes); por último retardo del crecimiento intrauterino con 0,07 (5 pacientes). De 6475 gestantes añosas, el 3,72\% (241 pacientes) presentaron sufrimiento fetal agudo, el 3,46\% (224 pacientes) presentó sepsis del recién nacido; en tercer lugar hipoglicemia del recién nacido con un $0,23 \%$ (15 pacientes); óbito fetal con $0.14 \%$ (9 pacientes); y con un valor mínimo 0,05\% (3 pacientes) [Tabla 3 y 4 ].

Se realizó la asociación entre la edad materna y las complicaciones maternas, y encontramos que el valor de significancia es 0,01 (menor a 0,05 ) por lo que concluimos que la edad materna influye en la presencia de complicaciones maternas.

Al existir asociación entre las variables, se observa que, con una confianza del $95 \%$ el OR: 1,08 , es decir que al haber tenido edad materna mayor o igual a 35 años aumenta en 0.08 más el riesgo de padecer complicaciones

Tabla 4. Complicaciones perinatales en la gestante adolescente

\begin{tabular}{|l|r|r|}
\hline \multicolumn{1}{|c|}{ COMPLICACIONES PERINATALES } & FRECUENCIA & PORCENTAJE \\
\hline SIN COMPLICACIONES & 7086 & 92.69 \\
\hline SUFRIMIENTO FETAL AGUDO & 368 & 4.81 \\
\hline SEPSIS DEL RECIEN NACIDO & 160 & 2.09 \\
\hline & & \\
\hline HIPOGLICEMIA DEL RECIEN NACIDO & 13 & 0.17 \\
\hline OBITO FETAL & 13 & 0.17 \\
\hline $\begin{array}{l}\text { RETARDO DE CRECIMIENTO } \\
\text { INTRAUTERINO }\end{array}$ & & \\
\hline & 5 & 0.07 \\
\hline
\end{tabular}

añosa

\begin{tabular}{|l|r|r|}
\hline COMPLICACIONES PERINATALES & FRECUENCI & \multicolumn{1}{l|}{ A } \\
\hline SIN COMPLICACIONES & 5983 & \multicolumn{1}{l|}{ PORCENTAJE } \\
\hline SUFRIMIENTO FETAL AGUDO & 241 & 92.40 \\
\hline SEPSIS DEL RECIEN NACIDO & 224 & 3.72 \\
\hline & & 3.46 \\
\hline HIPOGLICEMIA DEL RECIEN NACIDO & 15 & 0.23 \\
\hline OBITO FETAL & 9 & 0.14 \\
\hline RETARDO DE CRECIMIENTO & & \\
INTRAUTERINO & 3 & 0.05 \\
\hline Total & 6475 & 100.00 \\
\hline
\end{tabular}


Tabla 5. Asociación entre edad materna y morbilidad materna.

\begin{tabular}{lcc}
\hline Edad Materna & OR (IC95\%) & p valor \\
\hline Gestante Adolescente & 1 & \\
Gestante Añosa & $1.08(1.02-1.15)$ & 0.01 \\
\hline
\end{tabular}

Fuente. Ficha de Recolección de datos.

maternas en comparación con la gestante adolescente (Tabla 5).

Se realizó también la asociación entre la edad materna y la las complicaciones perinatales, y encontramos que el valor de significancia es 0.15 (mayor a 0.05 ) por lo que concluimos que la edad materna no influye en la presencia de complicaciones perinatales.

Al realizar la asociación mediante la Prueba Chi cuadrado entre cada patología materna y la edad materna, solo mostraron asociación tres patologías.

Se presenta la asociación entre la edad materna y la amenaza de aborto y encontramos que el valor de la prueba Chi Cuadrado arroja un valor de significancia de 0,006 (menor a 0,05) por lo que concluimos que el tener una edad mayor o igual que 35 años influye en la presencia de amenaza de aborto durante el embarazo. Al existir asociación se observa que, con una confianza del 95\% el OR: 3,6, es decir que las gestantes en edad materna avanzada presenta 2,6 más veces de padecer amenaza de aborto que la gestante adolescente (Tabla 6). Al realizar la asociación entre la edad materna y placenta

Tabla 6. Asociación entre edad materna y amenaza de aborto.

\begin{tabular}{lcc}
\hline & OR (IC95\%) & p valor \\
\hline Gestante Adolescente & 1 & \\
Gestante Añosa & $3.6(1.43-9.09)$ & 0.006 \\
\hline
\end{tabular}

Fuente. Ficha de Recolección de datos.

previa, se encontró que el valor de la prueba Chi Cuadrado arroja un valor de significancia de 0,000 (menor a 0,05 ) por lo que concluimos que el tener una edad mayor o igual que 35 años influye en la gestante en padecer placenta previa. Al existir asociación se observa que, con una confianza del $95 \%$ el OR: 13,9 , es decir que las gestantes en edad materna avanzada presenta 12,9 más veces de padecer placenta previa que la gestante adolescente (Tabla 7).

Tabla 6. Asociación entre edad materna y placenta previa.

\begin{tabular}{lcc}
\hline & OR (IC95\%) & p valor \\
\hline Gestante Adolescente & 1 & \\
Gestante Añosa & $13.90(6.44-30.12)$ & 0.00 \\
\hline
\end{tabular}

Fuente. Ficha de Recolección de datos.
Tabla 8. Asociación entre edad materna y preclampsia

\begin{tabular}{lcc}
\hline & OR (IC95\%) & p valor \\
\hline Gestante Adolescente & 1 & \\
Gestante Añosa & $1.54(1.48-1.92)$ & 0.00 \\
\hline
\end{tabular}

Fuente. Ficha de Recolección de datos.

Se presenta la asociación entre la edad materna y preeclampsia, y encontramos que el valor de la prueba Chi Cuadrado arroja un valor de significancia de 0,000 (menor a 0,05 ) por lo que concluimos que el tener una edad mayor o igual que 35 años influye en la gestante en padecer preeclampsia durante el embarazo. Al existir asociación se observa que, con una confianza del $95 \%$ el OR: 1,54, es decir que las gestantes en edad materna avanzada presenta 1,54 más veces de padecer preeclampsia que la gestante adolescente (Tabla 8).

\section{DISCUSIÓN}

Nuestro estudio evidenció que las patologías más frecuentes en madres de edad avanzada y adolescentes fueron la pre eclampsia, anemia y ruptura prematura de membranas; en las gestantes añosas la preeclampsia se presentó en un $4,65 \%$ vs $3,10 \%$ en adolescentes coincidiendo con el estudio de Caicedo y Ahumada $^{5}$ donde determina que la preeclampsia es más frecuente en la gestante añosa. Por otro lado anemia se presentó en un $4,51 \%$ para la gestante adolescente y $4,62 \%$ para la gestante añosa, predominando esta patología en la gestante añosa y difiriendo del mismo estudio ya que en adolescentes se presentó en un 7,5\% en adolescentes y un $6,3 \%$ en añosas. En cuanto a ruptura prematura de membranas en el presente estudio se presentó en un porcentaje de $6,33 \%$ para adolescentes y $4,57 \%$ para las gestantes añosas coincidiendo también con el Caicedo y Ahumada donde se encontró que la ruptura prematura de membranas predomino en la gestante adolescente con $18,8 \%$ vs $15 \%$ en la gestante añosa. En complicaciones perinatales nuestro estudio arrojó que las adolescentes presentaron mayor porcentaje de complicaciones perinatales en comparación con la gestante añosa, óbito fetal se presentó en un $0,17 \%$ en adolescentes vs $0,14 \%$ en añosas; sufrimiento fetal agudo con $4,81 \%$ y $3.72 \%$ en adolescentes y añosas, respectivamente. Por ultimo RCIU se presentó en $0,07 \%$ en gestantes adolescente $y$ en $0,05 \%$ en gestantes añosas, coincidiendo con los autores Caicedo y Ahumada ya que en su estudio las gestantes adolescentes presentan mayor frecuencia de complicación perinatal.

La presente investigación arrojo que el grupo etario de gestantes con más riesgo de complicarse son las madres con edad avanzada (de 35 años o más), con un 0,08 veces más de riesgo que las adolescentes; resultado que difiere con Caicedo y Ahumada quienes determinaron que el grupo de más riesgo al comparar ambas poblaciones fueron las adolescentes con un 1,5 veces más de riesgo. 
No se encontró que la edad materna sea factor de riesgo para complicaciones perinatales al igual que en el estudio de Rivas ${ }^{8}$.

No se encontraron estudios similares donde se determine la asociación y riesgo de presentar alguna complicación materno perinatal en la gestante de edad materna avanzada en comparación con la gestante adolescente, en el presente estudio se encontró que las tres morbilidades que se asocian a la edad materna avanzada son, amenaza de aborto con OR: 3,6 ( $p=0,006)$, placenta previa OR:13,09 $(p=0,000)$, preeclampsia con un OR: $1,54(p=0,000)$.

\section{CONCLUSIONES}

Las patologías más frecuentes para la gestante adolescente fue ruptura prematura de membranas con $6,33 \%$ seguida de anemia con $4,51 \%$, en tercer lugar preeclampsia con $3,10 \%$, infecciones del tracto urinario en un $2,21 \%$ y distocia de presentación con $1,20 \%$; y en gestantes añosas en primer lugar se presentó preeclampsia en un $4,65 \%$, anemia con $4,62 \%$, en tercer lugar ruptura prematura de membranas con $4,57 \%$, infecciones del tractoo urinario $2,30 \%$ y placenta previa con $1,27 \%$. En complicaciones perinatales en madres adolescentes sufrimiento fetal agudo en un $4,81 \%$, sepsis del recién nacido en $2,09 \%$, hipoglicemia del recién nacido $0,17 \%$, óbito fetal $0,17 \%$ y restricción de crecimiento intrauterino en $0,05 \%$; por otro lado en las gestantes añosas el sufrimiento fetal fue de 2,09\%, sepsis del recién nacido en un 2,09\%, hipoglicemia del recién nacido $0,14 \%$ y restricción de crecimiento intrauterino en $0,05 \%$.

Se determinó que la gestante de edad materna avanzada presenta mayor riesgo de complicación materna $(0,08$ veces más riesgo) que las gestantes adolescentes.

Se determinó que las patologías que presentan asociación con edad materna avanzada fueron: amenaza de aborto(OR:3,06 $p=0,006)$, placenta previa (OR:13,09 $p=0,000)$ y preeclampsia (OR:1,54 $p=0,000)$.

\section{RECOMENDACIONES}

Los trastornos hipertensivos, anemia predominan en ambos grupos, hoy en día la preeclampsia sigue siendo una de las principales causas de mortalidad materna, se recomienda un buen manejo oportuno y seguimiento adecuado mediante controles prenatales periódicos, ya que al tener un diagnóstico temprano se puede evitar un compromiso mayor en la madre y el hijo.

La gestante adolescente al igual que la gestante añosa es considerada de alto riesgo, en el presente estudio se demostró que la gestante añosa presenta mayor riesgo de complicaciones materna, por ello se recomienda crear

un servicio de gestantes añosas para e incluir programas orientados a la maternidad en esta edad extrema.

Se recomienda monitoreo intensivo, informar y concientizar a la gestante con el riesgo que presenta al cursar con una edad materna avanzada; en cuanto a amenaza de aborto y

previa, que la gestante esté alerta ante la presencia de sangrado, dolor y acuda a un centro salud; en la paciente con preeclampsia se recomienda monitoreo doppler entre la semana 11 y $14 ; 20$ y 26 semanas por la implicancia clínica que presenta.

Financiamiento: autofinanciado.

Conflictos de interés: los autores declaran no tener algún conflicto de interés.

\section{REFERENCIAS BIBLIOGRÁFICAS}

1. Donoso E. Mortalidad materna en Chile, 2000-2004. Rev Chil Obstet Ginecol 2006; 71(4): 246-251.

2. Chong-Pun B, Tsz-Hsi T. Effect of parity and advanced maternal age on obstetric outcome. International Journal of Gynecology and Obstetrics 2008; 102: 237-241.

3. Sánchez M, Muñoz A . Influencia de padres y amigos sobre la actitud hacia las conductas sexuales de prevención en la adolescencia. Un análisis en función del genero. Revista Latinoamericana de Psicología 20053771-79. Disponible en: http://www.redalyc. org/articulo.oa?id=80537104. Fecha de consulta: 20 de diciembre de 2016.

4. Rodríguez O. Es la edad materna avanzada un factor de riesgo independiente para complicaciones maternoperinatales. Per Ginecol Obstet .2006;5(2):179-85.

5. Caicedo $F$, Ahumada $F$, Bolaños $M$, Ortega $O$, Tejada C. Complicaciones maternas y neonatales en pacientes embarazadas adolescentes y mayores de 35. Hospital Universitario San José de Popayán, Colombia. Tesis de Especialidad. Cauca, Colombia, 2008. 12 Pp.

6. Instituto Nacional Materno Perinatal. Analisis de la Situación de los Servicios Hospitalarios del Instituto Nacional Materno Perinatal - Maternidad de Lima. Ministerio de Salud-Instituto de Gestión de Servicios de Salud. Disponible en: http://www. inmp.gob.pe/institucional/asis-ho/1421426743. Acceso: 20 de diciembre de 2016.

7. Tipiani O. ¿Es la edad materna avanzada un factor de riesgo independiente para complicaciones materno-perinatales? Rev Per Ginecol Obstet. 2006;52(3):89-99.

8. Rivas E. Asociación entre edad materna avanzada y los resultados perinatales adversos en una clínica universitaria de Cartagena (Colombia), 2012. Estudio de cohorte retrospectiva. Revista Colombiana de Obstetricia y Ginecología. 2015; 66:179-185.

\section{ORCID iDs}

Félix Dasio Ayala Peralta

Enrique Guevara Ríos

Antonio Luna Figueroa

Oscar Antonio Limay Ríos

\section{Correspondencia:}

Dirección: Jirón Maracaibo 2153- San Martín de Porres-Lima 31. Teléfono: 567-6501 Celular: 999227657

E-mail: fayala1401@hotmail.com 